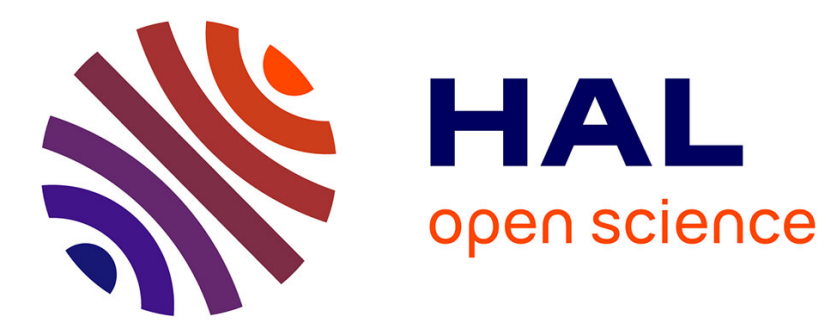

\title{
2D micro teleoperation with force feedback
}

\author{
Aude Bolopion, Barthélemy Cagneau, Stéphane Régnier
}

\section{To cite this version:}

Aude Bolopion, Barthélemy Cagneau, Stéphane Régnier. 2D micro teleoperation with force feedback. 2009 IEEE/RSJ International Conference on Intelligent Robots and Systems (IROS 2009), Oct 2009, St. Louis, MO, United States. pp.3265-3270, 10.1109/IROS.2009.5354778 . hal-02912205

\section{HAL Id: hal-02912205 https://hal.science/hal-02912205}

Submitted on 5 Aug 2020

HAL is a multi-disciplinary open access archive for the deposit and dissemination of scientific research documents, whether they are published or not. The documents may come from teaching and research institutions in France or abroad, or from public or private research centers.
L'archive ouverte pluridisciplinaire HAL, est destinée au dépôt et à la diffusion de documents scientifiques de niveau recherche, publiés ou non, émanant des établissements d'enseignement et de recherche français ou étrangers, des laboratoires publics ou privés. 


\title{
2D micro teleoperation with force feedback
}

\author{
Aude Bolopion, Barthélemy Cagneau and Stéphane Régnier
}

\begin{abstract}
This paper presents a 2D teleoperation task at microscales with force feedback. At this scale, two major problems arise while performing manipulation tasks: the lack of real time visual feedback, and the difficulty to determine the interaction forces. Therefore, indications must be provided to help the user perform a given task. In this paper, we provide the user with intuitive force feedback, to improve objects' manipulation using a haptic device. Our platform is composed of a tipless beam manipulator, which is deformed when forces are applied to it. These deformations are measured using a laser. The force information we provide to the user is based on the raw beam's deformation measurement, and mechanical properties of the probe. It does provide the operator with indications about the interaction forces.

This approach is validated by performing lateral and longitudinal rolling operations using microspheres with a radius of 25-micrometers. 2D rolling telemanipulation at microscale with force feedback is successfully demonstrated.
\end{abstract}

\section{INTRODUCTION}

During the last decades, the importance of micro and nano robotic systems has increased dramatically with the development of MEMS and micro assembly. However, handling of the components (objects of micrometer or nanometer sizes) remains a major problem [1]. Several manipulation tools exist, including micromanipulators with capacitive or piezoresistive sensors, micro tweezers and cantilevers. Among them, Scanning Probe Microscopes based manipulation provide the highest resolution for real time force sensing.

To perform manipulation tasks at micro or nanoscales, a major problem is the lack of real-time visual feedback. To overcome this difficulty, virtual reality (VR) is widely used [2]. The environment with which the operator interacts can be purely virtual (for teaching purposes, for example) [3], or based on periodic measurements made during an experiment [4]. Haptic feedback would also appear to be an interesting solution, since it adds the sense of touch during the manipulation. When based on direct measurements performed during the manipulation, it allows the user to receive information about the objects being manipulated [5]. It can also be used in addition to a VR system [6]. In this case, metaphors can be derived from the simulation, and be rendered as virtual guides to the operator. So far, manipulations with force feedback based on direct force measurement mainly consist in feeling or indenting objects [7] and characterizing them [8]. These operations are the first steps towards more complex tasks.

In this paper, we propose $2 \mathrm{D}$ telemanipulation with force feedback. We use a cantilever to manipulate objects, and

Authors are with Institut des Systèmes Intelligents et de Robotique, Université Pierre et Marie Curie, CNRS UMR 7222, 4 Place Jussieu, 75005 Paris, France $\{$ bolopion, cagneau, regnier\}@isir.fr measure the deformations of the beam using a laser reflected onto a photodiode. Using these measurements and mechanical properties of the probe, we determine the force applied on it by the object. This information is used to derive comprehensible indications about the manipulation that will be given to the user. Haptic feedback is chosen as the means to provide the user with the derived information. This approach is successfully demonstrated by microsphere rolling experiments.

This paper is organized as follows: In section II, forces available from the measurement of a cantilever's deformations are detailed. They will be used in section III to derive comprehensible forces that will be sent to the user to help him or her perform rolling manipulations. Results are presented in section $\mathrm{V}$ using the material and the control scheme described in section IV.

\section{2D FORCE MEASUREMENT FOR HAPTIC FEEDBACK}

The manipulation platform is composed of a cantilever with no tip. As explained in detail in [9], this kind of probe can be used to manipulate objects. Their deformations are measured using a laser, reflected onto a photodiode (figure 1). Knowing the stiffnesses of the beams, forces applied on the probes are determined from the deformation measurements. Calibration methods of the cantilevers' spring constants can be found in particular in [10] or [11].

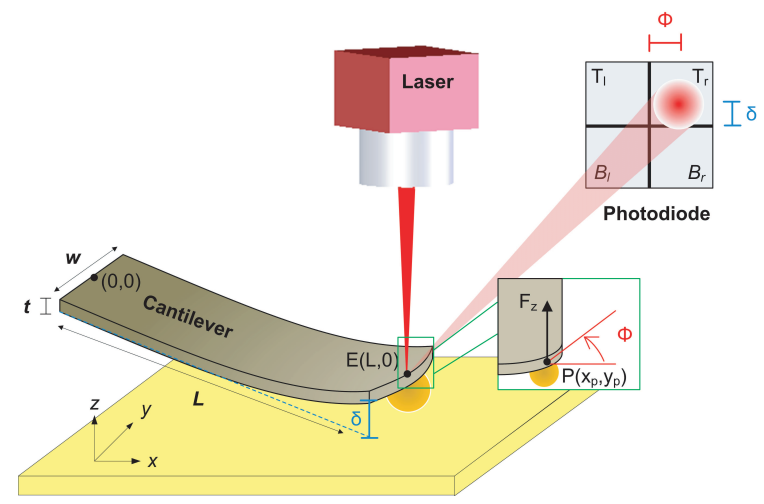

Fig. 1. Principle of force measurement using a laser

\section{A. Measurement of the cantilever's deformations}

The photodiode is composed of four different parts $\left(T_{l}\right.$, $\left.T_{r}, B_{l}, B_{r}\right)$, as shown on figure 1. Two output voltages are defined, $u_{v}$ and $u_{h}$, that are computed as:

$$
\begin{aligned}
& u_{v}=\left(T_{l}+T_{r}\right)-\left(B_{l}+B_{r}\right) \\
& u_{h}=\left(T_{l}+B_{l}\right)-\left(T_{r}+B_{r}\right)
\end{aligned}
$$


They correspond respectively to a displacement of the laser beam's impact along the $y$ and $z$ axes. To estimate the deformations of the cantilever, the vertical and horizontal sensitivities of the photodiode $\left(S_{v}\right.$ and $\left.S_{h}\right)$ need to be calibrated. Since the laser beam is focused in point $E$ situated in the middle line and at the extremity of the probe (see figure 1), only the cantilever's deformations at point $E$ can be measured. The deflection $\delta(L, 0)$, and angle of rotation $\phi(L, 0)$ are measured:

$$
\begin{aligned}
\delta(L, 0) & =S_{v} u_{v} \\
\phi(L, 0) & =S_{h} u_{h}
\end{aligned}
$$

B. Relation between the cantilever's deformations and the forces

The AFM cantilever is a simple beam the length, width and thickness of which will be noted respectively $L, w$, and $t$. All the parameters related to the cantilever are summarized in table I. Numerical values are given for a silicon beam, and the dimensions are those of the cantilever used in section $\mathrm{V}$ for the experiments.

TABLE I

VARIABLES DESCRIBING THE AFM PROBE, AND RELATED NUMERICAL VALUES (FOR A SILICON CANTILEVER). DIMENSIONS GIVEN ARE THE ONES OF THE CANTILEVER USED IN SECTION V)

\begin{tabular}{|c|c||c|}
\cline { 2 - 3 } \multicolumn{1}{c|}{} & \multicolumn{1}{c|}{ Signification } & Numerical values \\
\hline$L, t, w$ & $\begin{array}{c}\text { ength, thickness and } \\
\text { width of the } \\
\text { cantilever }\end{array}$ & $\begin{array}{c}L=220 \mu m, t=2.9 \mu m, \\
w=27 \mu m\end{array}$ \\
\hline$k_{z}, k_{t_{\phi}}$ & $\begin{array}{c}\text { normal stiffness and } \\
\text { torsional spring of } \\
\text { the cantilever }\end{array}$ & $\begin{array}{c}k_{z}(L)=2.4 N \cdot m^{-1}, \\
k_{t_{\phi}}(L)=64.0 \cdot 10^{-9} N . m\end{array}$ \\
\hline$E, G$ & $\begin{array}{c}\text { resp. Young's } \\
\text { modulus and the } \\
\text { shear modulus }\end{array}$ & $E=150 G P a, G=\frac{E}{2(1+\nu)}$ \\
\hline$\nu$ & $\begin{array}{c}\text { Poisson's ratio } \\
\text { area about the } x \\
\text { (resp. } y \text { ) axis }\end{array}$ & $I_{x}=\frac{t w^{3}}{12}, I_{y}=\frac{t^{3} w}{12}$ \\
\hline$I_{x}, I_{y}$ & \begin{tabular}{c} 
second moments of \\
\hline
\end{tabular} \\
\hline
\end{tabular}

As a force $F_{z}\left(x_{p}, y_{p}\right)$ is applied on the beam at point $P$ $\left(x_{p}, y_{p}\right)$ along the vertical direction ( $z$ axis), the cantilever will experience two different deformations, deflection and rotation. In the following paragraphs, we will derive relations between the applied force and the deformations of the probe.

1) Deformations along the $x$ axis: The force $F_{z}\left(x_{p}, y_{p}\right)$ bends the beam. Considering a clamp-free cantilever, the deflection of the probe $\delta\left(x, y_{p}\right)$ is given by:

$$
\delta\left(x, y_{p}\right)=\frac{F_{z}\left(x_{p}, y_{p}\right)}{E I_{y}}\left(x \frac{x_{p}^{2}}{2}-\frac{x_{p}^{3}}{6}\right) \text { for } x_{p} \leq x \leq L
$$

As seen in section II-A, this bending can be measured using equation (2). Therefore, the force $F_{z}\left(x_{p}, y_{p}\right)$ can be estimated using this measurement:

$$
F_{z}\left(x_{p}, y_{p}\right)=k_{z}\left(x_{p}\right) \delta\left(x_{p}, y_{p}\right)
$$

where $k_{z}\left(x_{p}\right)$ is the stiffness of the cantilever at point $x_{p}$ :

$$
k_{z}\left(x_{p}\right)=\frac{E w t^{3}}{4 x_{p}^{3}}=\left(\frac{L}{x_{p}}\right)^{3} k_{z}(L)
$$

The normal stiffness at the extremity of the cantilever $k_{z}(L)$ is calibrated. According to (4):

$$
\delta\left(x_{p}, y_{p}\right)=\frac{2 x_{p}}{3 L-x_{p}} \delta\left(L, y_{p}\right)
$$

Combining equations (6) and (7) leads to:

$$
F_{z}\left(x_{p}, y_{p}\right)=\frac{2 L^{3}}{x_{p}^{2}\left(3 L-x_{p}\right)} k_{z}(L) \delta\left(L, y_{p}\right)
$$

2) Deformations along the $y$ axis: Deformation is derived considering a cantilever along the $y$ axis (free at its extremity), and the hypothesis that the problem is monodimensional. The force $F_{z}\left(x_{p}, y_{p}\right)$ applied in the vertical direction produces a rotation $\phi\left(x_{p}, y_{p}\right)$ of the beam:

$$
\phi\left(x_{p}, y_{p}\right)=\frac{F_{z}\left(x_{p}, y_{p}\right)}{2 E I_{x}} y_{p}^{2}+\phi\left(x_{p}, 0\right)
$$

This angle can be used to compute the corresponding moment $M\left(x_{p}, y_{p}\right)$ :

$$
M\left(x_{p}, y_{p}\right)=k_{t_{\phi}}\left(x_{p}\right) \phi\left(x_{p}, y_{p}\right)
$$

where $k_{t_{\phi}}\left(x_{p}\right)=\frac{G w t^{3}}{3 x_{p}}$ is the torsional spring constant. According to (9), this leads to:

$$
M\left(x_{p}, y_{p}\right)=k_{t_{\phi}}\left(x_{p}\right)\left[\frac{F_{z}\left(x_{p}, y_{p}\right)}{2 E I_{x}} y_{p}^{2}+\phi\left(x_{p}, 0\right)\right]
$$

The relations between the force applied on the cantilever and the measure of its deformations presented in this section will be used in the following paragraphs to derive comprehensible information about the interaction forces, to help the user perform a given manipulation task.

\section{AN EXAMPLE OF 2D MANIPULATION WITH FORCE FEEDBACK}

The use of haptic feedback greatly improves the dexterity of an operator while performing a manipulation task in the microworld [12]. This is especially true when the visual feedback is limited, which is the case at microscales since optical microscopes provide only two dimensional images. A rolling experiment has been chosen to demonstrate the use of force feedback for micro manipulation. This is a promising way to manipulate objects (spheres, carbon nanotubes) at this scale. It can also be used to release objects attached to a cantilever [9], or to perform patterning on a substrate [13]. It has to be noted that other strategies of manipulation could have been chosen, as well as different object sizes. Rolling is only one possible application to demonstrate how to render interaction forces to the operator using haptic feedback.

\section{A. Longitudinal rolling}

In [9], the authors state that to make objects roll without sliding, a force similar to the pull-off force must be applied on them by the cantilever. Since the cantilever is not in the plane $(x, O, y)$, but tilted by several degrees (see figure 1) the deflection at the extremity of the cantilever will be kept constant to apply this force. The position of the origin of 
the cantilever will therefore be servoed to maintain it at the desired value.

The initial position of the sphere under the cantilever is noted $x_{p}(0)$. This point, as well as the position of the cantilever along the $x$ axis $x_{c}(j)$ at time step $j$, are presumed to be known (see paragraph IV-A). Since rolling without sliding is performed, the current position of the sphere $x_{p}$ at time $j$ is estimated from:

$$
x_{p}(j)=x_{p}(0)+\frac{x_{c}(j)-x_{c}(0)}{2}
$$

For longitudinal rolling, the sphere is initially positioned at the extremity of the probe, in its middle line (point $E$ $\left(x_{\max }, 0\right)$ on figure 1$)$. The position along the $y$ axis is kept constant and is such that the cantilever experiences no torsion: $\phi(L, 0)=0$. The cantilever is then moved along the $x$ axis, to make the sphere roll. To provide the user with information about the position of the object being manipulated a force $F_{e}^{x}$ will be sent to him or her. This force is derived from the normal force $F_{z}$ applied by the sphere on the cantilever. However, since $\delta\left(L, y_{p}\right)$ is not available, we chose to send to the user a force $F_{e}^{x}$ computed as:

$$
F_{e}^{x}\left(x_{p}, y_{p}\right)=\frac{2 L^{3}}{x_{p}^{2}\left(3 L-x_{p}\right)} k_{z}(L) \delta(L, 0)
$$

where $\delta(L, 0)$ is measured using the photodiode (see (2)). The profile of the force is plotted on figure 2 (as stated above, the position of the origin of the cantilever is servoed so that $\delta(L, 0)$ is constant).

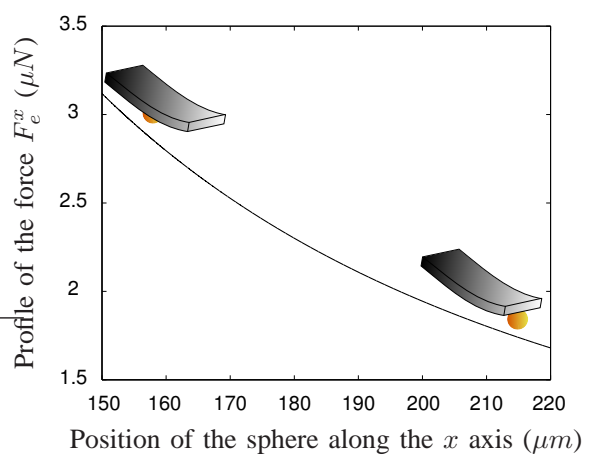

Fig. 2. Profile of the force that will be sent to the user along the $x$ axis

This force is minimal at the extremity of the cantilever. So that this point represents a stable equilibrium position for the user, an offset will be used, in order to render a force equal to zero when the sphere is at the extremity of the cantilever.

\section{B. Lateral rolling}

For lateral rolling, the position along the $x$ axis is kept constant, while the cantilever is moved along the $y$ axis so that the sphere rolls. While performing rolling tasks, it is important to keep the sphere in the middle line of the cantilever, so that it does not loose contact on a lateral side of the beam. To help the user maintain the sphere on the middle line, a force $F_{e}^{y}$ is sent to him or her via the haptic interface. It will correspond to the torsion force that will tend to keep the cantilever horizontal:

$$
\frac{M\left(x_{p}, y_{p}\right)}{y_{p}}
$$

However, according to (11), the variation of this force is not easily interpretable. Therefore, we chose to linearize the expression:

$$
F_{e}^{y}\left(x_{p}, y_{p}\right)=\frac{M(L, 0)}{\frac{w}{2}}=\frac{k_{t_{\phi}}(L)}{\frac{w}{2}} \phi(L, 0)
$$

where $\phi(L, 0)$ is the measure of the rotation given by the photodiode output (see equation (3)). The variations of the force $F_{e}^{y}$ are plotted on figure 3 .

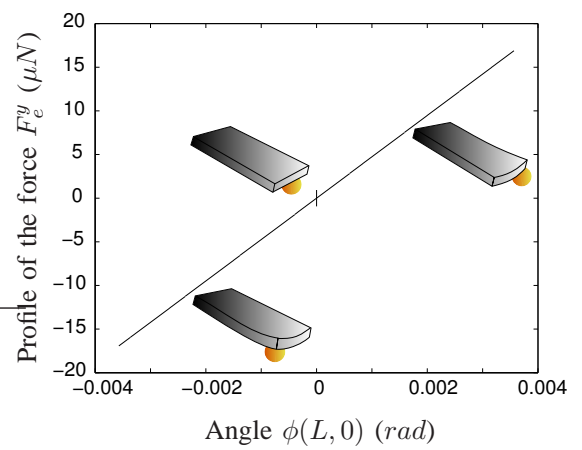

Fig. 3. Profile of the force that will be sent to the user along the $y$ axis

It can be seen that depending on the sign of $\phi(L, 0)$, this force is either positive or negative. The higher $\phi(L, 0)$ is, the higher the magnitude of the force. If sent back to the user through a haptic interface, this force will indeed tend to keep the sphere on the middle line of the cantilever (which is the point where the torsion is nil). Since the angle increases the further the force $F_{z}\left(x_{p}, y_{p}\right)$ is situated from the middle line, and $F_{e}^{y}$ increases as the angle increases, it can also help to estimate the position of the sphere $\left(x_{p}, y_{p}\right)$ under the cantilever.

\section{C. $2 D$ force feedback rolling operation}

As can be seen from (13) and (15), the forces $F_{e}^{x}$ and $F_{e}^{y}$ sent to the user are not coupled. Therefore, 2D rolling experiments can be carried out using the same force feedback rendered on two different axes of the haptic interface.

\section{EXPERIMENTAL SETUP}

\section{A. Platform}

The manipulation platform used to perform the experiments described in section III is depicted on figure 4(a).

The vision system is composed of an optical microscope with a $20 \times$ lens. The initial contact point between the sphere and the probe has been determined using this vision system.

To position the cantilever accurately with respect to the scene, three nanotranslators are available to manipulate objects along the $x, y$ and $z$ directions. The range (resp. resolution) provided by this system is equal to $50 \mu \mathrm{m}$ (resp. $0.1 \mathrm{~nm}$ ) for the $x$ and $y$ directions, $12 \mu \mathrm{m}$ (resp. $1.83 \mathrm{~nm}$ ) 

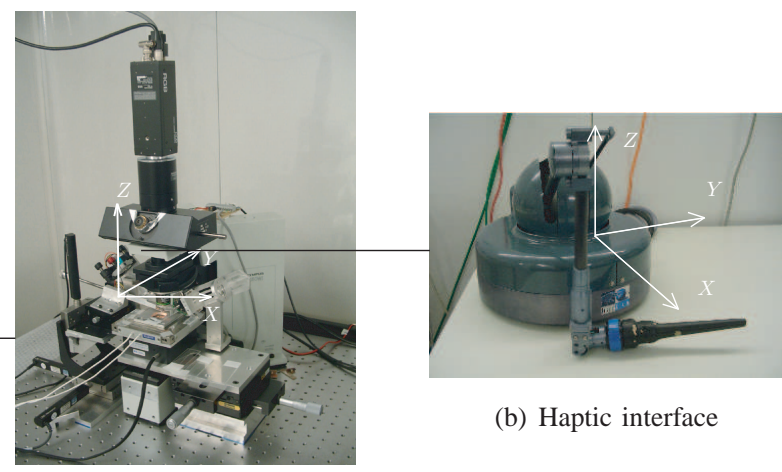

(b) Haptic interface

(a) General view of the platform

Fig. 4. Experimental setup

for the $z$ direction. At each time step, the position of the cantilever is known from the nanotranslators' sensors.

The available haptic device is a Virtuose manufactured by Haption ${ }^{1}$. This arm, represented on figure 4(b), allows 3 degrees of freedom of force feedback. For ease of manipulation, and to avoid damaging the master device, forces sent back to the user are saturated to $6 N$.

\section{B. Control scheme}

The choice of the control scheme used to connect the haptic device to the slave device is of primary importance for the quality of the rendering. [14] provides a detailed analysis of two different couplings. It concludes that among these two control schemes the Force-Position $(F P)$ is the one that leads to the best results for performing manipulation tasks at micro or nanoscales. It is depicted on figure 5. Scalar variables are used, since the two forces $F_{e}^{x}$ and $F_{e}^{y}$ are not coupled (see equations (13) and (15)), and will be rendered on two different axes of the haptic interface. The parameter $i$ is for $x$ or $y$, depending on the considered axis.

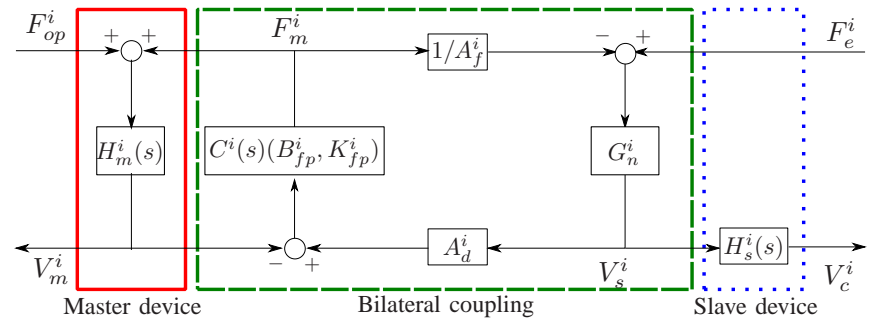

Fig. 5. Force-Position control scheme used to control the cantilever and provide force feedback. The $i$ parameter is for the variable $x$ or $y$, depending on the considered axis

The operator applies a force $F_{o p}^{i}$ on the haptic device handle, the velocity of which is $V_{m}^{i}$. This velocity will be used in the coupling to compute the desired velocity of the nanotranslator $\left(V_{s}^{i}\right)$. The actual velocity of the cantilever with respect to the substrate is $V_{c}^{i} . F_{e}^{i}$ represents either $F_{e}^{x}$ or $F_{e}^{y}$ computed as shown in equations (13) and (15). The force fed back to the user $F_{m}^{i}$ is computed using this variable.

\footnotetext{
${ }^{1} \mathrm{http}: / /$ www.haption.com
}

For each axis, the haptic device's continuous time transfer function $H_{m}^{i}(s)$ has been identified as being composed of inertia $M_{v}^{i}$ and damping $B_{v}^{i}$. The slave's $H_{s}^{i}(s)$ is a second order transfer function, with two time constants $\tau_{1}^{i}$ and $\tau_{2}^{i}$ :

$$
\begin{aligned}
H_{m}^{i}(s) & =\frac{1}{B_{v}^{i}+M_{v}^{i} s} \\
H_{s}^{i}(s) & =\frac{1}{\left(1+\tau_{1}^{i} s\right)\left(1+\tau_{2}^{i} s\right)}
\end{aligned}
$$

Due to scale differences between micro and macro worlds, scaling factors have to be used to enhance the forces fed back to the user (by a factor $A_{f}^{i}$ ), and scale down his or her motions (by a factor $A_{d}^{i}$ ). For stability reasons, two controllers are added. The first one is $C^{i}(s)$, a proportionalintegral $(P I)$ controller:

$$
C^{i}(s)=B_{f p}^{i}+\frac{K_{f p}^{i}}{s}
$$

It is used to compute the forces reflected to the user. The integral term determines the stiffness of the feeling, while the proportional term adds viscosity into the system and stabilizes it. The second controller is $G_{n}^{i}$, a proportional $(P)$ term, used to control the slave device.

Tuning of this coupling is important for the quality of the force rendering. $A_{f}^{i}$ and $A_{d}^{i}$ are determined by force amplification and precision of manipulation requirements. The range of admissible values for the gains of the $(P I)$ and $(P)$ controllers is derived taking into account the stability and transparency conditions.

In the following section the control scheme's parameters will be tuned according to table II [14]. $T_{e}$ (the sampling period) is equal to $5 \mathrm{~ms}$ for our system.

TABLE II

VALID RANGE OF VALUES FOR THE GAINS OF THE $(P I)$ AND $(P)$ CONTROLLERS.

\begin{tabular}{|c|c||c|}
\cline { 2 - 3 } \multicolumn{1}{c|}{} & Minimum value & Maximum value \\
\hline$K_{f p}$ & $\begin{array}{c}\text { high for a stiff contact } \\
\text { feeling }\end{array}$ & $\begin{array}{c}\frac{4 M_{v}}{T_{e}^{2}}, \text { low for } \\
\text { non-contact transparency }\end{array}$ \\
\hline$B_{f p}$ & $\frac{K_{f p} T_{e}}{2}-B_{v}$ & $\begin{array}{c}\text { low for non-contact } \\
\text { transparency }\end{array}$ \\
\hline$G_{n}$ & $\begin{array}{c}\text { high for non-contact } \\
\text { transparency }\end{array}$ & $\frac{A_{f}}{A_{d} K_{f p} \frac{T_{e}}{2}+A_{d} B_{f p}}$ \\
\hline
\end{tabular}

\section{EXPERIMENTAL RESULTS}

The strategy described in section III will be evaluated with rolling experiments using glass spheres with a radius of $25 \mu \mathrm{m}$.

\section{A. Longitudinal rolling}

In this section, a rolling experiment along the $x$ axis of the cantilever is presented. The vertical position of the probe is servoed in order to maintain deflection at its extremity constant $(\delta(L, 0)=0.7 \mu \mathrm{m}$, which corresponds to a force of $1.7 \mu \mathrm{N}$ when the sphere is at point $E$ ).

The controller gain coefficients are chosen according to table II. $A_{d}^{x}=50 \cdot 10^{3}$ is such that the displacement of the 
sphere is precise enough for the experiment. $A_{f}^{x}$ is chosen so that the force $F_{m}^{x}$ is on the order of a few newtons so that it can be easily felt by the user. In the case of longitudinal rolling, the force will be amplified by a factor $25 \cdot 10^{6} . K_{f p}^{x}=$ $100 N . m^{-1}$ and $B_{f p}^{x}=0.1 N . s . m^{-1}$ are chosen to allow a stiff feeling, while avoiding oscillations by adding some viscosity. $G_{n}^{x}$ is computed using the values of the other gains.

During the experiment, the user makes the sphere roll from the point $x=x_{\max }=L$ (extremity of the cantilever) to a point closer to the origin of the cantilever (noted $x_{m i n}$, and then back to a point situated between $x_{\min }$ and $x_{\max }$, noted $x_{\text {int }}$ (for $x$ intermediate). The user makes small oscillations by moving the sphere back and forth around this position $x_{\text {int }}$.

The results are plotted on figure 6 . The position of the sphere along the $x$ axis, as well as the forces sent to the operator $F_{m}^{x}$ are represented.

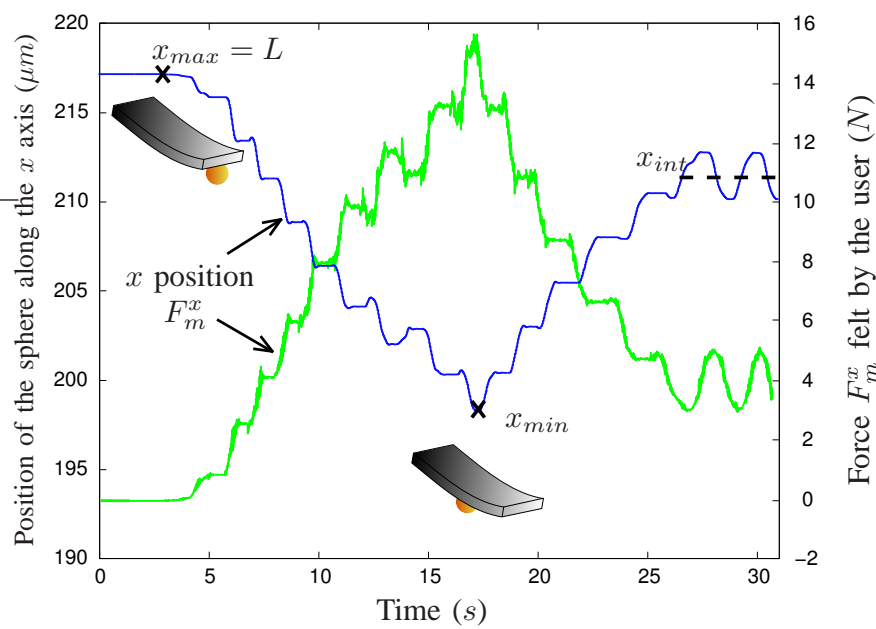

Fig. 6. Positions and forces during the longitudinal rolling experiment. Parameters used: $A_{f}^{x}=25 \cdot 10^{6}, A_{d}^{x}=50 \cdot 10^{3}, K_{f p}^{x}=100 N . m^{-1}$, $B_{f p}^{x}=0.1 N . s \cdot m^{-1}, G_{n}^{x}=825 m \cdot N^{-1} \cdot s^{-1}$

According to the velocity scaling factor $A_{d}^{x}$ used, a displacement of $1 \mathrm{~cm}$ in the haptic workspace corresponds to a displacement of $0.2 \mu \mathrm{m}$ for the cantilever. During this experiment, the probe is moved by around $40 \mu \mathrm{m}$, which implies a displacement of $2 m$ of the haptic handle. Due to the limited workspace of the master device (around $35 \mathrm{~cm}$ for the $x$ axis), a function to reset the position of the haptic device without moving the probe is used. The steps that can be seen on the plot of the position along the $x$ axis are due to these instants when the user resets the position of the haptic device (and therefore keeps the position of the sphere constant). As a consequence, the force sent back to the user is constant during this operation.

As shown by the results on figure 6 , and as predicted in section III-A (figure 2), the force sent to the user increases as the sphere gets further from the extremity of the cantilever. For $x=x_{\max }$, the force is nil. The operator has to make an effort to move the sphere further from this equilibrium position. The force felt by the user also helps him or her to evaluate how far the sphere is from point $x=x_{\max }$, as the magnitude of the force increases.

\section{B. Lateral rolling}

As in the case of longitudinal rolling, the probe's vertical deflection is kept constant by servoing the vertical position of the origin of the cantilever. The $x$ position is also kept constant.

The velocity scaling factor is the same as that used for lateral rolling (the desired precision of the manipulation is the same for both axes). Due to the expression of the force $F_{e}^{y}$ that has been chosen (moment transformed as a force), its magnitude is around 10 times higher than $F_{e}^{x}$. Since the same magnitude as $F_{m}^{x}$ is required for $F_{m}^{y}$ (so that it can be felt by the user and rendered through the haptic interface), the force scaling factor has been decreased by a factor 10 .

Concerning the PI controller, the same integral gain as for the $x$ axis is used. However, the proportional gain has to be increased, such that $B_{f p}^{y}=1.5$ N.s. $m^{-1}$. This is due to the variation between positive and negative forces that arise when the sphere moves from the left to the right side of the cantilever, and vice-versa. To avoid oscillations, viscosity must be added.

As for lateral rolling, $G_{n}^{y}$ is computed according to the value of the other gains. Due to the difference between the gains used for the $x$ and $y$ axes, the proportional gain of the $y$ axis is smaller than that for the $x$ axis.

The user manipulates the cantilever along the $y$ axis. Starting from the middle line of the cantilever $(y=0)$, he or she moves the sphere towards negative $y$ (phase 1). During phase 2 , he or she moves back to positive $y$. In phase 3 , he or she performs oscillations with the sphere, moving back and forth between positive and negative $y$.

The user feels the force $F_{m}^{y}$, computed from $F_{e}^{y}$ (see equation (15)). Results of both forces and positions along the $y$ axis are presented on figure 7 .

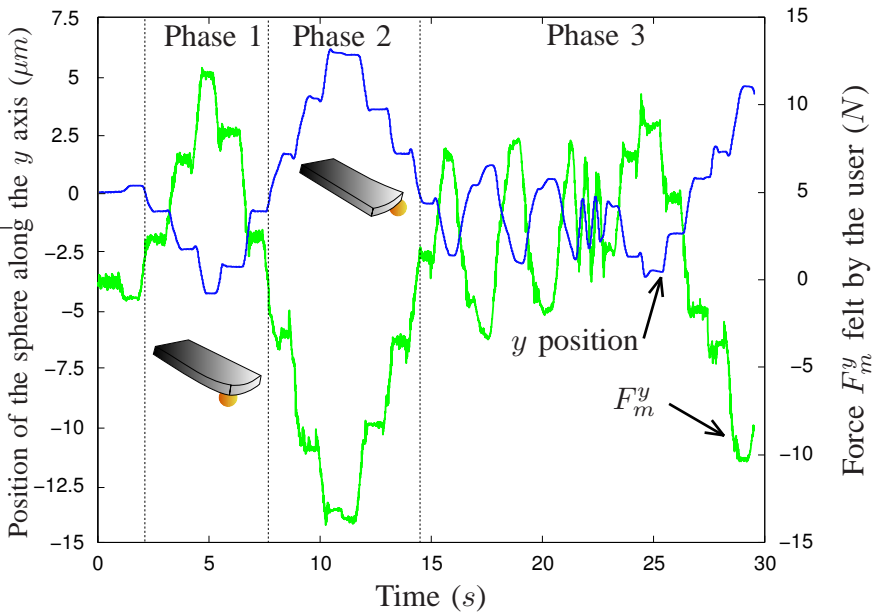

Fig. 7. Positions and forces during the lateral rolling experiment. Parameters used: $A_{f}^{y}=2 \cdot 10^{6}, A_{d}^{y}=50 \cdot 10^{3}, \quad K_{f p}^{y}=100 N . m^{-1}$, $B_{f p}^{y}=1.5 N . s . m^{-1}, G_{n}^{y}=19.8 m \cdot N^{-1} . s^{-1}$

The same velocity scaling factor as in section V-B is used $\left(A_{d}^{y}=50 \cdot 10^{3}\right)$. For the same reasons of limited 
haptic workspace (maximum $64 \mathrm{~cm}$ for the $y$ axis), the reset function is used, and produces the steps that can be seen on the plots of both positions and forces.

As stated in section III-B, the user feels a force that guide him or her to bring the cantilever into an equilibrium position (no torsion). The forces sent back allow an untrained user to feel distinctly on which side of the cantilever the sphere is positioned. Moreover, it has been verified experimentally that the system remains stable even if the user creates small oscillations (see plots at around $t=22 s$ ).

\section{C. $2 D$ Rolling}

This experiment consists in combining both lateral and longitudinal rolling, to perform a $2 \mathrm{D}$ rolling task with force feedback. This is possible since the signals used are independent: there is no coupling between the $F_{e}^{x}$ and $F_{e}^{y}$ forces computed. Therefore they can be rendered to the user using two axes of the haptic device.

As for lateral and longitudinal rolling the deflection $\delta(L, 0)$ is constant. The movement performed by the operator in the plane $(x O y)$ is depicted on figure 8 . The shape of the movement is roughly circular, but noticeable differences have been made to better distinguish the corresponding forces sent to the user. The movement has been repeated three times in order to verify that the forces felt by the operator are constant for a given position. The gains used are the same as those given in sections $\mathrm{V}-\mathrm{A}$ and $\mathrm{V}-\mathrm{B}$.

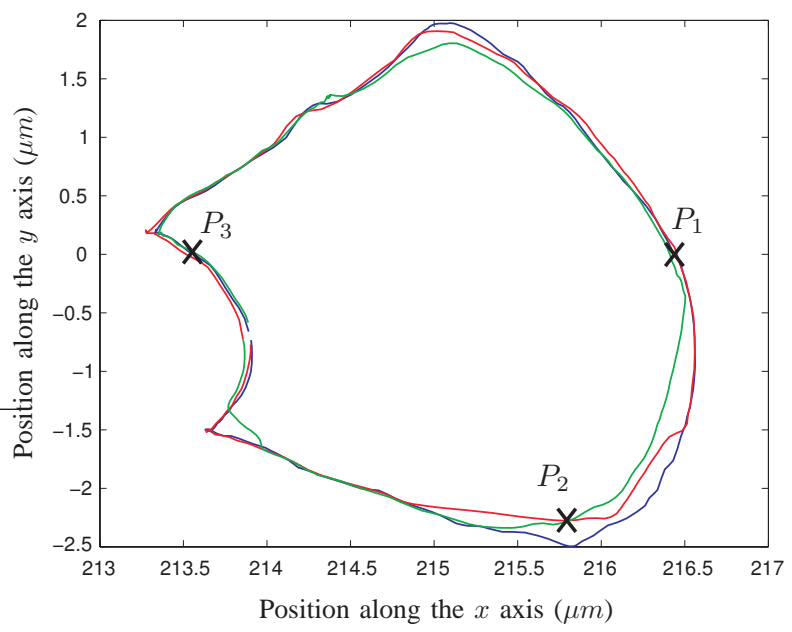

Fig. 8. Positions of the sphere on the plane $(x O y)$ during the 2D rolling experiment

Contrary to plots on figures 6 and 7, the positions along the $x$ and $y$ axes are not stepped. Since the displacements of the cantilever were small enough, it was not useful to use the haptic device's reset function.

The forces sent back to the user while performing this experiment are represented on figure 9, where the lateral force is plotted against the longitudinal force. Since the user chose to perform these circles without reaching the extremity of the cantilever, the force along the $x$ axis is never nil. For a given position, the operator feels the same force, as demonstrated by the three attempts and highlighted by the characteristic points noted $P_{1}, P_{2}$ and $P_{3}$. This helps the operator to estimate the position of the sphere under the cantilever.

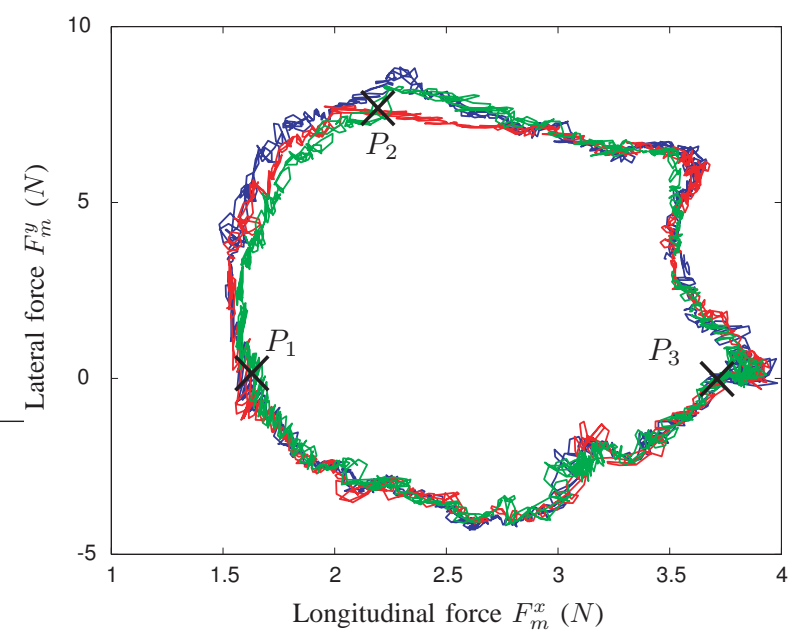

Fig. 9. Forces felt by the user on the $x$ and $y$ axes of the haptic interface during the $2 \mathrm{D}$ rolling experiment

During these experiments, the forces provide the user with comprehensible information, since he or she has to make an effort to get further from the defined equilibrium position (point $E$ ). This is possible thanks to the appropriate choice of the forces sent to the user, and a control scheme able to render these forces sufficiently amplified (so that the operator can feel them), while remaining stable. Using the same setup, other strategies to provide force information during a manipulation task can be considered.

\section{CONCLUSION}

In this paper, micromanipulation with force feedback using tipless cantilevers has been demonstrated. Forces that help the user bring objects to a desired position were derived from raw measurements of the cantilever's deformations, and the mechanical properties of the beams. These indications were provided to the user through a haptic interface, using a force position control scheme. The structure of this coupling, as well as the tuning of the gains, allowed for stable and transparent manipulation.

This approach has been validated using rolling experiments on 25 micrometer radius spheres. As shown experimentally for both longitudinal and lateral rolling, attractive forces indeed helped the user since he or she had to make an effort to move the sphere further from a defined equilibrium position. 2D rolling has also been demonstrated. Based on the magnitude and the direction of the forces felt, the user was also able to estimate the position of the sphere under the cantilever.

This work was the first step toward more complex teleoperations. An analysis to determine the most relevant profile of the forces sent to the user should be carried out. The possibility of using other strategies to manipulate objects via a haptic interface should also be studied. 


\section{ACKNOWLEDGMENT}

This work was supported by the French National Agency of Research, through the PACMAN project.

\section{REFERENCES}

[1] M. Sitti, "Microscale and nanoscale robotics systems [grand challenges of robotics]," IEEE Robotics and Automation Magazine, vol. 14, no. 1, pp. 53-60, March 2007.

[2] A. Ferreira and C. Mavroidis, "Virtual reality and haptics for nanorobotics," IEEE Robotics and Automation Magazine, vol. 13, no. 3, pp. 78-92, Sept. 2006.

[3] S.-G. Kim and M. Sitti, "Task-based and stable telenanomanipulation in a nanoscale virtual environment," IEEE Transactions on Automation Science and Engineering, vol. 3, no. 3, pp. 240-247, July 2006.

[4] G. Li, N. Xi, M. Yu, and W.-K. Fung, "Development of augmented reality system for AFM-based nanomanipulation," IEEE/ASME Transactions on Mechatronics, vol. 9, no. 2, pp. 358-365, June 2004.

[5] X. Tian, L. Liu, N. Jiao, Y. Wang, Z. Dong, and N. Xi, "3D nano forces sensing for an AFM based nanomanipulator," in Proceedings of the International Conference on Information Acquisition, June 2004, pp. 208-212.

[6] M. Ammi and A. Ferreira, "Robotic assisted micromanipulation system using virtual fixtures and metaphors," in Proceedings of the IEEE International Conference on Robotics and Automation, 10-14 April 2007, pp. 454-460.

[7] M. Sitti and H. Hashimoto, "Teleoperated touch feedback from the surfaces at the nanoscale: modeling and experiments," IEEE/ASME Transactions on Mechatronics, vol. 8, no. 2, pp. 287-298, June 2003.

[8] M. Guthold, M. Falvo, W. Matthews, S. Paulson, J. Mullin, S. Lord, D. Erie, S. Washburn, R. Superfine, F. Brooks Jr., and R. Taylor II, "Investigation and modification of molecular structures with the nanomanipulator," Journal of Molecular Graphics and Modelling, vol. 17, no. 3-4, pp. 187-197, 1999.

[9] D. S. Haliyo, F. Dionnet, and S. Régnier, "Controlled rolling of micro objects for autonomous micro manipulation," International Journal of Micromechatronics, vol. 3, no. 2, pp. 75-101, 2006.

[10] E. D. Langlois, G. A. Shaw, J. A. Kramar, J. R. Pratt, and D. C. Hurley, "Spring constant calibration of atomic force microscopy cantilevers with a piezosensor transfer standard," Review of Scientific Instruments, vol. 78, no. 9, p. 093705, 2007.

[11] R. G. Cain, S. Biggs, and N. W. Page, "Force calibration in lateral force microscopy," Journal of Colloid and Interface Science, vol. 227, pp. 55-65, 2000.

[12] G. Millet, A. Lécuyer, J. Burkhardt, S. Haliyo, and S. Régnier, "Improving perception and understanding of nanoscale phenomena using haptics and visual analogy," in Eurohaptics'08, M. Ferre (Ed.). Springer-Verlag, 2008, pp. 847-856.

[13] A. Tafazzoli, C. Pawashe, and M. Sitti, "Force-controlled microcontact printing using microassembled particle templates," May 2006, pp. $263-268$.

[14] A. Bolopion, B. Cagneau, D. S. Haliyo, and S. Régnier, "Tuning the gains of haptic couplings to improve force feedback stability in nanorobotics," To be published in Proceedings of the IEEE International Conference on Robotics and Automation, May 2009. 